\title{
COMMENT \\ Healthy mouth, healthy gut: a dysbiotic oral microbiome exacerbates colitis
}

\author{
N. M. Moutsopoulos (iD) and J. E. Konkel ${ }^{2,3}$ \\ Mucosal Immunology (2020) 13:852-854; https://doi.org/10.1038/s41385-020-00341-y
}

The oral mucosa is a site of first encounters for the immune system. ${ }^{1}$ Here commensal microbiota, airborne particles, food, and innocuous antigens are encountered prior to entry to the gastrointestinal tract. How these first encounters control the development and education of the immune system locally is not well understood. Importantly, whether immune responses generated in the oral mucosal environment will affect systemic or distal immunity in health and disease is not detailed to date.

Immune responses within the oral mucosa are strictly controlled, with dysregulated immunity frequently resulting in the development of the oral mucosal disease Periodontitis. This is a prevalent disease in which a dysbiotic microbiome colonizing the tooth triggers an exaggerated inflammatory response and consequent destruction of tooth supporting structures. For decades this disease has been associated clinically with various extraoral inflammatory diseases, including (but not limited to) cardiovascular disease, diabetes, Alzheimer's disease, rheumatoid arthritis and colitis. ${ }^{2,3}$ Although inflammatory mediators, as well as the dysbiotic oral microbiome associated with periodontitis, have been linked with the triggering and/or exacerbation of these distal pathologies, the exact mechanisms by which periodontitis contributes to the severity of these extraoral disease is unclear.

Recently, an elegant study by Kamada and colleagues provides detailed insight into the mechanisms by which Periodontitis enhances gut immunopathology during colitis (Fig. 1). ${ }^{4}$ The authors demonstrate that induction of experimental periodontitis in mice led to increased severity of DSS-induced colitis. Kitamoto et al. show that the oral pathobionts that outgrow in biomass and relative abundance during periodontitis aggravate gastrointestinal pathology via two distinct, but potentially interlinked, mechanisms. Firstly, specific oral pathobionts are able to colonize the colitic gut and enhance IL-1 $\beta$ production. Alongside this, the authors demonstrate that oral pathobiont reactive Th17 cells, primed in oral mucosa-draining lymph nodes, trafficked to the gut and became reactivated by periodontal microbiota traveling to the gastrointestinal tract through ingestion. This paper provides the first evidence that $T$ cells primed at the oral mucosal barrier traffic to distal sites, become reactivated and contribute to extraoral inflammatory pathology. These findings are significant from a translational standpoint, outlining immunological and microbiological links between the oral and gastrointestinal mucosa and mechanistically delineating how periodontitis promotes colitic inflammation. Importantly, this work also establishes the concept that immune cells educated at the oral mucosa, in this case T cells specific for oral pathobionts, can become re-activated systemically and affect distal immunity.

This work stimulates a plethora of further questions. From a basic science standpoint it would be important to establish whether $\mathrm{T}$ cell responses to oral commensals are induced at the oral barrier during health, or whether these are only induced in periodontitis when breach of the barrier allows for the local immune system to come into close contact with a greater burden of microbiota. Interestingly, while Th17 responses in the gingival oral mucosa are triggered in response to periodontal pathobionts in disease, in the setting of health Th17 cells have been shown to expand at the gingiva independent of live microbiota and in response to mechanical damage from mastication..$^{5-7}$ Along the same lines, whether oral commensal microbiota in health promote largely tolerogenic responses but periodontal pathobionts are uniquely equipped to trigger pathogenic responses, is also not well appreciated. Specifically, the microbial elements that favor Th17 induction by periodontal pathogens are not well understood. One, non-bacterial, oral commensal well-appreciated for its ability to drive IL-17-mediated immunity is the fungal oral commensal Candida albicans. ${ }^{8}$ Whether oral-induced Candidaspecific Th17 cells become re-activated at distal sites and participate in systemic immunity to Candida is not yet recognized.

In addition to induction of oral-pathobiont-specific Th17 cells, how else might periodontitis impact gastrointestinal immune homeostasis? Interestingly, this paper also briefly highlights other possible immunological mediators of an oral-gastrointestinal cross-talk. Specifically, alterations in TCRy $\delta$ T cells and B cells are also seen in the gut of animals with periodontitis. Do these key mediators of the host-microbial dialog also contribute to periodontitis-induced exacerbation of colititc pathology or are they altered as a result of changes to the gastrointestinal commensal community in mice with oral inflammation?

Previous work has indicated that oral commensal bacteria can translocate and colonize the gut, where they can contribute to the development of both inflammatory colitis and cancerous pathology, specifically Klebsiella spp..$^{9}$ and Fusobacterium. ${ }^{10}$ However, constituents of the oral microbiota are not routinely found in the gut, so how are these, and other, oral commensals able to colonize, bloom and persist within the gastrointestinal environment? Oral pathobionts will bypass the colonization resistance that they typically have in the gastrointestinal tract when they find a favorable ecological niche; such a niche could exist in the setting of already established gut inflammation. In fact, gut inflammation

TOral Immunity and Inflammation Section, National Institute of Dental and Craniofacial Research, NIH, Bethesda, MD 20892, USA; ${ }^{2}$ Faculty of Biology, Medicine and Health,

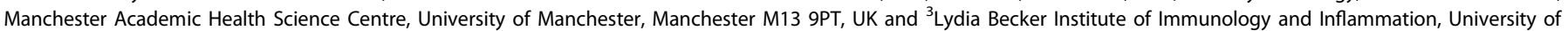
Manchester, Manchester M13 9NT, UK

Correspondence: N M. Moutsopoulos (nmoutsop@mail.nih.gov) or J E. Konkel (joanne.konkel@manchester.ac.uk)

Received: 30 June 2020 Revised: 30 July 2020 Accepted: 12 August 2020

Published online: 25 August 2020 


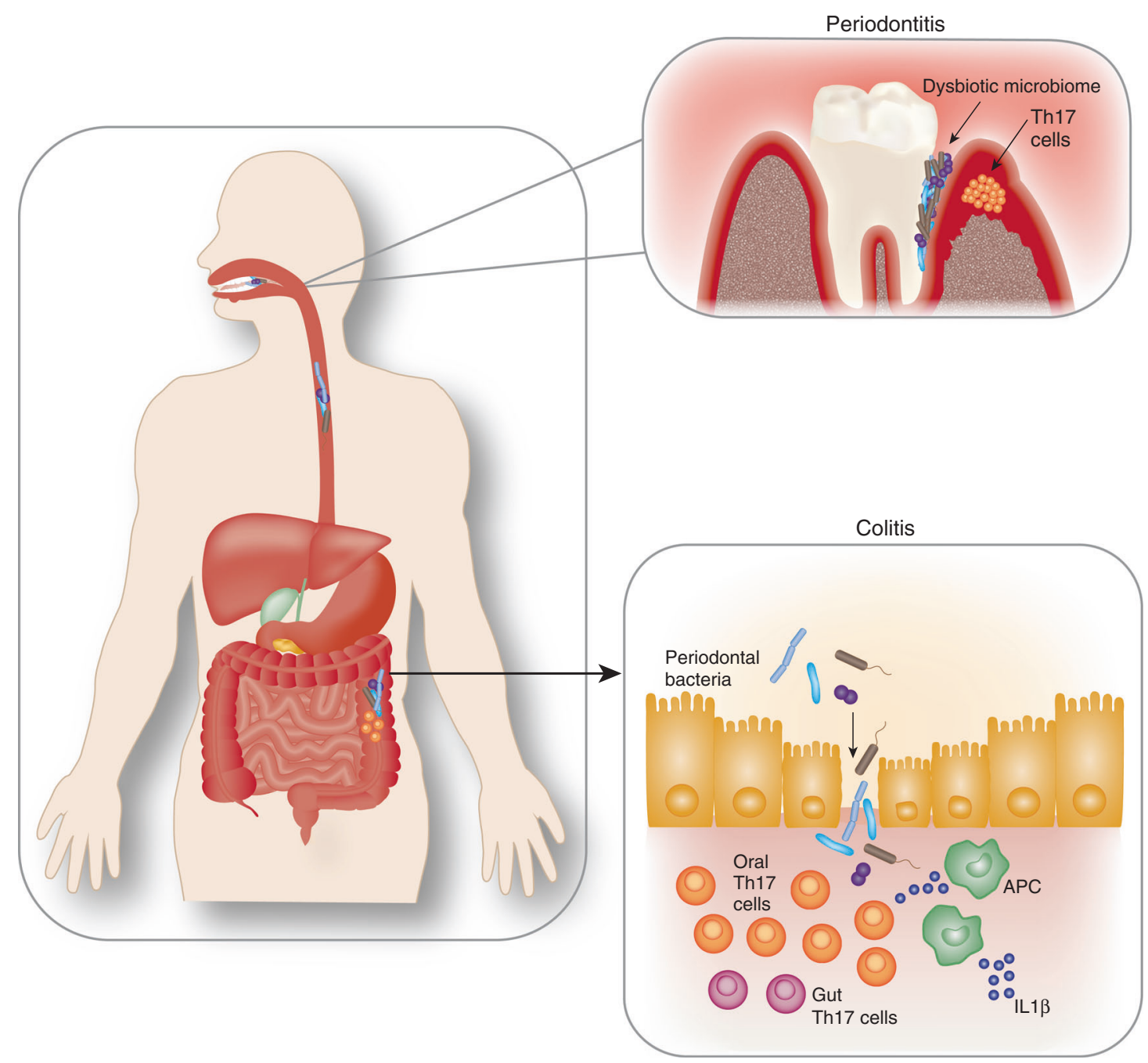

Fig. 1 Immune and microbial cross-talk between the oral and gastrointestinal mucosa. Recent work form Kamada and colleague's support a link between the oral disease periodontitis with elevated immunopathology in colitis (hypothetic model shown in humans). The authors demonstrate that periodontal pathobionts from the oral cavity, through ingestion, colonize the colitic gut and aggravate disease. Critical in this process is also the induction of oral pathobiont-reactive Th17 cells which are educated in the oral mucosa and subsequently traffic to the gut. These oral Th17 cells become reactivated in the gut by oral pathobionts and exacerbate colitic pathology.

favors colonization of oral pathobionts such as Enterobacteriaceae (for example Klebsiella spp.), which are metabolically adapted to thrive in settings of inflammation. While Enterobacteriaceae are not abundant in human periodontitis, human periodontal pathogens also thrive in inflammatory conditions ${ }^{3}$ and thus this concept would translate to settings of human periodontitis. Additionally, this, and previous studies, ${ }^{9}$ would also suggest that gastrointestinal colonization by oral pathobionts is enabled by a genetic susceptibility to colitis, which promotes dysbiosis in the intestinal microbiome prior to pathology. Given the multi-factorial drivers of colitis, it would be interesting to consider whether other colitic risk factors, including diet and early-life use of antibiotics, would promote the colonization of oral pathobionts in the gastrointestinal tract and whether the co-existence of periodontitis with such risk factors may predispose to more severe and/or an earlier onset of disease. However, whether oral commensals initiate and/or aggravate gastrointestinal inflammation in individuals already at risk of developing colitic pathology will be difficult to decipher.

Whether periodontal-pathobionts are able to colonize other sites and/or whether immune cells reactive to these bacteria contribute to the pathology of other diseases eagerly awaits investigation. Periodontal pathobionts are not only ingested into the gastrointestinal tract but also translocate into the systemic circulation through the vasculature. Dissecting whether periodontal pathobiont-reactive immune cells traffic to and participate in the lesions of other inflammatory diseases linked to periodontitis should now be readily explored.

Finally, it is important to consider the potential translational implications of this work. Understanding the genetic and environmental determinants that allow gut colonization of oral pathobionts might be key to stratifying patient groups in which presence of periodontitis is a significant risk factor for the development of gastrointestinal inflammatory disease. Beyond risk assessment and personalized prevention, one can conceivably speculate that this work opens up the possibility to investigate novel biomarkers and therapeutic interventions in colitis. Whether gut colonization with specific oral-pathobionts can serve as a disease biomarker for colitis, remains to be explored. Similarly, targeting oral pathobionts in the treatment and/or prevention of colitic inflammation may also be an important therapeutic avenue to consider.

While the clinical and therapeutic potential of this work can only be speculative at this point, findings from this study clearly demonstrate novel concepts and mechanisms by which oral 
inflammation and oral microbes are connected to gastrointestinal disease. This work should stimulate and provide a platform for future work investigating the immunological connections between the oral and gastrointestinal mucosa in both health and disease.

\section{ACKNOWLEDGEMENTS}

This work was funded in part by the intramural program of the National Institute of Dental and Craniofacial Research, NIH (to N.M.M.) by the BBSRC [BB/M025977/1], Versus Arthritis, and Lister Institute (all to J.E.K.).

\section{AUTHOR CONTRIBUTIONS}

N.M.M. and J.E.K. conceived of and wrote this commentary.

\section{ADDITIONAL INFORMATION}

Competing interests: The authors declare no competing interests.

Publisher's note Springer Nature remains neutral with regard to jurisdictional claims in published maps and institutional affiliations.

\section{REFERENCES}

1. Moutsopoulos, N. M. \& Konkel, J. E. Tissue-specific immunity at the oral mucosal barrier. Trends Immunol. 39, 276-287 (2018).

2. Konkel, J. E., O'Boyle, C. \& Krishnan, S. Distal consequences of oral inflammation. Front. Immunol. 10, 1403 (2019).

3. Hajishengallis, G. Periodontitis: from microbial immune subversion to systemic inflammation. Nat. Rev. Immunol. 15, 30-44 (2015).

4. Kitamoto, S. N.-K. et al. The intermucosal connection between the mouth and gut in commensal pathobiont-driven colitis. Cell 182, 447-462 (2020).

5. Dutzan, N. et al. On-going mechanical damage from mastication drives homeostatic Th17 cell responses at the oral barrier. Immunity 46, 133-147 (2017).

6. Dutzan, N. et al. A dysbiotic microbiome triggers TH17 cells to mediate oral mucosal immunopathology in mice and humans. Sci. Transl. Med. 10, eaat0797 (2018).

7. Tsukasaki, M. et al. Host defense against oral microbiota by bone-damaging T cells. Nat. Commun. 9, 701 (2018).

8. Gaffen, S. L. \& Moutsopoulos, N. M. Regulation of host-microbe interactions at oral mucosal barriers by type 17 immunity. Sci. Immunol. 5, eaau4594 (2020).

9. Atarashi, K. et al. Ectopic colonization of oral bacteria in the intestine drives $\mathrm{TH} 1$ cell induction and inflammation. Science 358, 359-365 (2017).

10. Kostic, A. D. et al. Genomic analysis identifies association of Fusobacterium with colorectal carcinoma. Genome Res. 22, 292-298 (2012). 